\title{
Necessary and sufficient conditions for symmetric homogeneous polynomial inequalities of degree four and six in real variables
}

Vasile Cirtoaje

Department of Automatic Control and Computers, University of Ploiesti, Ploiesti, Romania.

Dedicated to George A Anastassiou on the occasion of his sixtieth birthday

Communicated by Professor G. Sadeghi

\begin{abstract}
Let $f_{n}(x, y, z)$ be a symmetric homogeneous polynomial of degree $n=4$ or $n=6$, in three real variables. We give necessary and sufficient conditions to have $f_{n}(x, y, z) \geq 0$ for all real numbers $x, y, z$. Then, we apply the obtained results to prove several relevant symmetric homogeneous polynomial inequalities of degree four and six.
\end{abstract}

Keywords: Symmetric homogeneous polynomial; necessary and sufficient conditions; real variables. 2010 MSC: Primary 26B25.

\section{Introduction}

A symmetric and homogeneous polynomial of degree four in three variables $x, y, z$ can be written as

$$
f_{4}(x, y, z)=A_{1} \sum x^{4}+A_{2} \sum x y\left(x^{2}+y^{2}\right)+A_{3} \sum x^{2} y^{2}+A_{4} x y z \sum x,
$$

where $A_{1}, A_{2}, A_{3}, A_{4}$ are real constants, and $\sum$ denotes a cyclic sum over $x, y, z$. Using the notations

$$
p=x+y+z, \quad q=x y+y z+z x, \quad r=x y z,
$$

${ }^{*}$ Corresponding author

Email address: vcirtoaje@upg-ploiesti.ro (Vasile Cirtoaje) 
by the known identities

$$
\begin{gathered}
\sum x^{4}=p^{4}-4 p^{2} q+2 q^{2}+4 p r, \\
\sum x y\left(x^{2}+y^{2}\right)=p^{2} q-2 q^{2}-p r, \\
\sum x^{2} y^{2}=q^{2}-2 p r,
\end{gathered}
$$

we can write $f_{4}(x, y, z)$ in the form

$$
f_{4}(x, y, z)=A r+B p^{4}+C p^{2} q+D q^{2},
$$

where $A, B, C, D$ are real constants.

The best known fourth degree symmetric homogeneous polynomial inequality in three real variables is no doubt Schur's inequality, which states that

$$
\sum x^{2}(x-y)(x-z) \geq 0,
$$

or, equivalently,

$$
\sum x^{4}+x y z \sum x \geq \sum x y\left(x^{2}+y^{2}\right) .
$$

The following generalization of the fourth degree Schur's inequality was proved in [2].

Proposition 1.1. Let $\alpha$ and $\beta$ be real numbers. The inequality

$$
\sum x^{4}+\beta \sum x^{2} y^{2}+(2 \alpha-\beta+1) x y z \sum x \geq(\alpha+1) \sum x y\left(x^{2}+y^{2}\right)
$$

holds for all real numbers $x, y, z$ if and only if $\beta \geq \alpha^{2}+2 \alpha$.

In the main particular case $\beta=\alpha^{2}+2 \alpha$, the inequality in Proposition 1.1 is equivalent to the elegant inequality ([3], pp. 77)

$$
\sum(x-y)(x-z)(x-\alpha y)(x-\alpha z) \geq 0,
$$

where equality holds for $x=y=z$, and for $x / \alpha=y=z$ (if $\alpha \neq 0$ ).

On the other hand, Tetsuya Ando proved in [1] the following fourth degree symmetric homogeneous polynomial inequality.

Proposition 1.2. If $\alpha$ and $x, y, z$ are real numbers, then

$$
\sum x^{4}+\left(\alpha^{2}+2\right) \sum x^{2} y^{2}+2 \alpha(\alpha-1) x y z \sum x \geq 2 \alpha \sum x y\left(x^{2}+y^{2}\right),
$$

with equality for $x^{2}+y^{2}+z^{2}=\alpha(x y+y z+z x)$.

A symmetric and homogeneous polynomial of degree six can be written as

$$
\begin{aligned}
& f_{6}(x, y, z)=A_{1} \sum x^{6}+A_{2} \sum x y\left(x^{4}+y^{4}\right)+A_{3} \sum x^{2} y^{2}\left(x^{2}+y^{2}\right) \\
& +A_{4} \sum x^{3} y^{3}+A_{5} x y z \sum x^{3}+A_{6} x y z \sum x y(x+y)+A_{7} x^{2} y^{2} z^{2},
\end{aligned}
$$

where $A_{1}, \ldots, A_{7}$ are real constants. Using the identities

$$
\begin{gathered}
\sum x^{6}=3 r^{2}+6\left(p^{3}-2 p q\right) r+p^{6}-6 p^{4} q+9 p^{2} q^{2}-2 q^{3}, \\
\sum x y\left(x^{4}+y^{4}\right)=-3 r^{2}+\left(7 p q-p^{3}\right) r+p^{4} q-4 p^{2} q^{2}+2 q^{3}, \\
\sum x^{2} y^{2}\left(x^{2}+y^{2}\right)=-3 r^{2}-2\left(p^{3}-2 p q\right) r+p^{2} q^{2}-2 q^{3},
\end{gathered}
$$




$$
\begin{gathered}
\sum x^{3} y^{3}=3 r^{2}-3 p q r+q^{3}, \\
\sum x^{3}=3 r+p^{3}-3 p q, \quad \sum x y(x+y)=p q-3 r
\end{gathered}
$$

$f_{6}(x, y, z)$ can be expressed in the form

$$
f_{6}(x, y, z)=A r^{2}+g_{1}(p, q) r+g_{2}(p, q),
$$

with

$$
g_{1}(p, q)=B p^{3}+C p q, \quad g_{2}(p, q)=D p^{6}+E p^{4} q+F p^{2} q^{2}+G q^{3},
$$

where $A, B, C, D, E, F, G$ are real constants. Throughout the paper, we call the coefficient $A$ of $r^{2}$ in the development (1.7) the highest coefficient of $f_{6}(x, y, z)$.

As we will show in the next section, the first step in proving the inequality $f_{6}(x, y, z) \geq 0$ using necessary and sufficient conditions is to write the sixth degree symmetric homogeneous polynomial $f_{6}(x, y, z)$ in the form (1.7). To make this, the following identities are also useful sometimes:

$$
\begin{gathered}
(x-y)^{2}(y-z)^{2}(z-x)^{2}=\sum x^{2} y^{2}\left(x^{2}+y^{2}\right)-2 \sum x^{3} y^{3} \\
-2 x y z \sum x^{3}+2 x y z \sum x y(x+y)-6 x^{2} y^{2} z^{2}, \\
(x-y)^{2}(y-z)^{2}(z-x)^{2}=-27 r^{2}+2\left(9 p q-2 p^{3}\right) r+p^{2} q^{2}-4 q^{3} .
\end{gathered}
$$

In our opinion, to prove sixth degree symmetric homogeneous polynomial inequalities using necessary and sufficient conditions is always possible, but rather complicated and tedious when the highest coefficient of $f_{6}(x, y, z)$ is positive. For this reason, it is beneficent to find out a suitable technique based on strong sufficient conditions for proving such inequalities in a simpler way, without making the development (1.7) in $p, q$ and $r$. On the other hand, the approach of the symmetric homogeneous polynomial inequalities with nonnegative real variables, using either necessary and sufficient conditions or only some strong sufficient conditions, is also an interesting and opportune work, already under our investigation.

Our proposed necessary and sufficient conditions for inequalities in real variables are presented in section 2 and proved in section 3. In section 4, we apply the obtained results for proving some relevant symmetric homogeneous polynomial inequalities of degree four and six. The last six applications are sixth degree inequalities having the highest coefficient positive, which were posted in 2009 and 2010 on the well-known website Art of Problem Solving ([4], [5], [6], 7]). Notice that no solution was given to these difficult inequalities.

\section{Main Results}

In order to prove our main results, we need the following lemma.

Lemma 2.1. Let $x \leq y \leq z$ be real numbers such that $x+y+z=p$ and $x y+y z+z x=q$, where $p$ and $q$ are given real numbers satisfying $p^{2} \geq 3 q$. The product

$$
r=x y z
$$

is minimal when $y=z$, and is maximal when $x=y$; that is,

$$
r \in\left[r_{\min }(p, q), r_{\max }(p, q)\right]
$$

where

$$
\begin{aligned}
& r_{\text {min }}(p, q)=\frac{\left(p-2 \sqrt{p^{2}-3 q}\right)\left(p+\sqrt{p^{2}-3 q}\right)^{2}}{27} \\
& r_{\text {max }}(p, q)=\frac{\left(p-\sqrt{p^{2}-3 q}\right)^{2}\left(p+2 \sqrt{p^{2}-3 q}\right)}{27} .
\end{aligned}
$$


Using Lemma 2.1, we can prove the following theorems.

Theorem 2.2. Let $f_{4}(x, y, z)$ be a fourth degree symmetric homogeneous polynomial. The inequality

$$
f_{4}(x, y, z) \geq 0
$$

holds for all real $x, y, z$ if and only if $f_{4}(x, 1,1) \geq 0$ and $f_{4}(x, 0,0) \geq 0$ for all real $x$.

Theorem 2.3. Let $f_{6}(x, y, z)$ be a sixth degree symmetric homogeneous polynomial which has its highest coefficient non-positive $(A \leq 0)$. The inequality

$$
f_{6}(x, y, z) \geq 0
$$

holds for all real $x, y, z$ if and only if $f_{6}(x, 1,1) \geq 0$ and $f_{6}(x, 0,0) \geq 0$ for all real $x$.

Notice that using Theorem 2.2 leads to a short solution for any fourth degree symmetric homogeneous polynomial inequality in real variables. For instant, with regards to Proposition 1.1, if we denote

$$
f_{4}(x, y, z)=\sum x^{4}+\beta \sum x^{2} y^{2}+(2 \alpha-\beta+1) x y z \sum x-(\alpha+1) \sum x y\left(x^{2}+y^{2}\right),
$$

then

$$
f_{4}(x, 1,1)=(x-1)^{2}\left[(x-\alpha)^{2}+\beta-\alpha^{2}-2 \alpha\right]
$$

and

$$
f_{4}(x, 0,0)=x^{4} .
$$

Thus, by Theorem 2.2, the conclusion follows. Also, with regards to Proposition 1.2, if we denote

$$
f_{4}(x, y, z)=\sum x^{4}+\left(\alpha^{2}+2\right) \sum x^{2} y^{2}+2 \alpha(\alpha-1) x y z \sum x-2 \alpha \sum x y\left(x^{2}+y^{2}\right),
$$

then

$$
f_{4}(x, 1,1)=\left(x^{2}-2 \alpha x+2-\alpha\right)^{2} \geq 0
$$

and

$$
f_{4}(x, 0,0)=x^{4} \geq 0 .
$$

With regard to the polynomial $f_{6}(x, y, z)$ written in the form

$$
f_{6}(x, y, z)=A r^{2}+g_{1}(p, q) r+g_{2}(p, q),
$$

where $p=x+y+z, q=x y+y z+z x, r=x y z$, let us denote

$$
\begin{gathered}
h_{1}(t)=2 A t+g_{1}(t+2,2 t+1), \\
h_{2}(t)=2 A t^{2}+g_{1}\left(1+2 t, 2 t+t^{2}\right), \\
d(p, q)=g_{1}^{2}(p, q)-4 A g_{2}(p, q) .
\end{gathered}
$$

In addition, assume that

$$
\begin{aligned}
d(t+2,2 t+1)>0 & \Longleftrightarrow t \in \Gamma_{1}, \\
d\left(1+2 t, 2 t+t^{2}\right)>0 & \Longleftrightarrow t \in \Gamma_{2} .
\end{aligned}
$$

Theorem 2.4. Let $f_{6}(x, y, z)$ be a sixth degree symmetric homogeneous polynomial having the highest coefficient $A>0$. Consider the following three conditions:

(a) $f_{6}(t, 1,1) \geq 0$ and $f_{6}(t, 0,0) \geq 0$ for all real $t$;

(b) $h_{1}(t) \geq 0$ for $t \in[-2,1] \cap \Gamma_{1}$

(c) $h_{2}(t) \leq 0$ for $t \in[-1 / 2,1] \cap \Gamma_{2}$.

The inequality $f_{6}(x, y, z) \geq 0$ holds for all real $x, y, z$ if and only if the condition (a) and one of the conditions (b) and (c) are fulfilled. 


\section{Proof of Lemma 2.1 and Theorems $2.2,2.3,2.4$}

Proof of Lemma 2.1. First, we show that $x \in\left[x_{1}, x_{2}\right]$, where

$$
x_{1}=\frac{p-2 \sqrt{p^{2}-3 q}}{3}, \quad x_{2}=\frac{p-\sqrt{p^{2}-3 q}}{3} .
$$

From

$$
\begin{aligned}
(y-z)^{2} & =(y+z)^{2}-4 y z=(y+z)^{2}+4 x(y+z)-4 q \\
& =(p-x)^{2}+4 x(p-x)-4 q=-3 x^{2}+2 p x+p^{2}-4 q \geq 0,
\end{aligned}
$$

we get $x \geq x_{1}$, with equality for $y=z$. Similarly, from

$$
(x-y)(x-z)=x^{2}-2 x(y+z)+q=x^{2}-2 x(p-x)+q=3 x^{2}-2 p x+q \geq 0,
$$

we get $x \leq x_{2}$, with equality for $x=y$.

On the other hand, from

$$
x y z=x[q-x(y+z)]=x q-x^{2}(p-x)=x^{3}-p x^{2}+q x,
$$

we get $r(x)=x^{3}-p x^{2}+q x$. Since $r^{\prime}(x)=3 x^{2}-2 p x+q=(x-y)(x-z) \geq 0, r(x)$ is increasing on $\left[x_{1}, x_{2}\right]$, and hence $r(x)$ is minimal for $x=x_{1}$, when $y=z$, and is maximal for $x=x_{2}$, when $x=y$. It is easy to check that $r$ is minimal for

$$
x=\frac{p-2 \sqrt{p^{2}-3 q}}{3}, y=z=\frac{p+\sqrt{p^{2}-3 q}}{3},
$$

and is maximal for

$$
x=y=\frac{p-\sqrt{p^{2}-3 q}}{3}, \quad z=\frac{p+2 \sqrt{p^{2}-3 q}}{3} .
$$

Proof of Theorem 2.2. Let $p=x+y+z, q=x y+y z+z x, r=x y z$. For fixed $p$ and $q$, the inequality $f_{4}(x, y, z) \geq 0$ can be written as $g(r) \geq 0$, where $g(r)$ is a linear function having the form (1.1). Since $g(r)$ is minimal when $r$ is minimal or maximal, by Lemma 2.1, it follows that $g(r)$ is minimal when two of $x, y, z$ are equal. Since the polynomial $f_{4}(x, y, z)$ is symmetric, homogeneous and satisfies $f_{4}(-x,-y,-z)=f_{4}(x, y, z)$, the conclusion follows.

Proof of Theorem 2.3. Let $p=x+y+z, q=x y+y z+z x, r=x y z$. For fixed $p$ and $q$, the inequality $f_{6}(x, y, z) \geq 0$ can be written as $g(r) \geq 0$, where $g(r)$ is a quadratic function having the form (1.7). Since $g(r)$ is concave for $A \leq 0$, it is minimal when $r$ is minimal or maximal. By Lemma 2.1, it follows that $g(r)$ is minimal when two of $x, y, z$ are equal. Since the polynomial $f_{6}(x, y, z)$ is symmetric, homogeneous and satisfies $f_{6}(-x,-y,-z)=f_{4}(x, y, z)$, the conclusion follows.

Proof of Theorem 2.4. Since $d(p, q)$ is the discriminant of the quadratic function

$$
g(r)=A r^{2}+g_{1}(p, q) r+g_{2}(p, q), \quad A>0,
$$

the desired inequality $g(r) \geq 0$ holds if $d(p, q) \leq 0$. Consider further that $d(p, q)>0$. By Lemma 2.1, for fixed $p$ and $q, r$ attains its extreme values when two of $x, y, z$ are equal. Then, the necessary conditions $g\left(r_{\min }\right) \geq 0$ and $g\left(r_{\max }\right) \geq 0$ are satisfied if the necessary conditions in (a) are fulfilled. In addition, 
the inequality $g(r) \geq 0$ holds for all real numbers $x, y, z$ if and only if either $r_{\min }(p, q) \geq \frac{-g_{1}(p, q)}{2 A}$, or $r_{\text {max }}(p, q) \leq \frac{-g_{1}(p, q)}{2 A} ;$ that is, either

$$
2 A r_{\min }(p, q)+g_{1}(p, q) \geq 0
$$

or

$$
2 A r_{\text {max }}(p, q)+g_{1}(p, q) \leq 0 .
$$

Due to the property $f_{6}(-x,-y,-z)=f_{6}(x, y, z)$, we may consider that $p=x+y+z \geq 0$. Since $d(p, q)=0$ for $p=q=0$, assume further that $p^{2}+q^{2} \neq 0, p \geq 0$. The following two cases generate the conditions in (b) and (c), respectively.

Case 1. $2 A r_{\min }(p, q)+g_{1}(p, q) \geq 0$.

We will show that the desired inequality holds if the conditions (a) and (b) are fulfilled. Let us denote

$$
a=\frac{p-2 \sqrt{p^{2}-3 q}}{3}, \quad b=\frac{p+\sqrt{p^{2}-3 q}}{3}>0, \quad t=\frac{a}{b} .
$$

It is easy to check that

$$
p=a+2 b, \quad q=2 a b+b^{2} .
$$

From $a \leq b, b>0$, and $p=a+2 b=b(t+2) \geq 0$, we get $-2 \leq t \leq 1$. By Lemma 2.1, we can write the condition $2 A r_{\min }(p, q)+g_{1}(p, q) \geq 0$ as

$$
2 A a b^{2}+g_{1}\left(a+2 b, 2 a b+b^{2}\right) \geq 0 .
$$

Dividing by $b^{3}$, we obtain $h_{1}(t) \geq 0$. In addition, we can write the condition $d(p, q)>0$ as $d\left(a+2 b, 2 a b+b^{2}\right)>$ 0 . Dividing by $b^{6}$, we get $d(t+2,2 t+1)>0$, which is equivalent to $t \in \Gamma_{1}$. Thus, the conclusion follows.

Case 2. $2 A r_{\text {max }}(p, q)+g_{1}(p, q) \leq 0$.

We will show that the desired inequality holds if the conditions (a) and (c) are fulfilled. Let

$$
a=\frac{p-\sqrt{p^{2}-3 q}}{3}, \quad b=\frac{p+2 \sqrt{p^{2}-3 q}}{3}, \quad t=\frac{a}{b} .
$$

It is easy to check that

$$
p=2 a+b, \quad q=a^{2}+2 a b .
$$

From $a \leq b, b>0$, and $p=2 a+b=b(2 t+1) \geq 0$, we get $-1 / 2 \leq t \leq 1$. By Lemma 2.1, we can write the condition $2 A r_{\max }(p, q)+g_{1}(p, q) \leq 0$ as

$$
2 A a^{2} b+g_{1}\left(2 a+b, a^{2}+2 a b\right) \leq 0 .
$$

Dividing by $b^{3}$, we obtain $h_{2}(t) \leq 0$. In addition, we can write the condition $d(p, q)>0$ as $d\left(2 a+b, a^{2}+2 a b\right)>$ 0 . Dividing by $b^{6}$, we get $d\left(1+2 t, 2 t+t^{2}\right)>0$, which is equivalent to $t \in \Gamma_{2}$. This completes the proof.

\section{Applications}

We will prove one inequality of fourth degree and ten inequalities of sixth degree, the last six of them having the highest coefficient positive. Notice that the coefficient of the product $(x-y)^{2}(y-z)^{2}(z-x)^{2}$ in these six inequalities has the best values.

Proposition 4.1. If $x, y, z$ are real numbers, then

$$
10 \sum x^{4}+64 \sum x^{2} y^{2} \geq 33 \sum x y\left(x^{2}+y^{2}\right),
$$

with equality for $x / 3=y=z$ [8]. 
Proof. By Theorem 2.2, it suffices to prove that $f_{4}(x, 1,1) \geq 0$ and $f_{4}(x, 0,0) \geq 0$ for all real $x$, where

$$
f_{4}(x, y, z)=10 \sum x^{4}+64 \sum x^{2} y^{2}-33 \sum x y\left(x^{2}+y^{2}\right) .
$$

We have

$$
f_{4}(x, 1,1)=2(x-3)^{2}\left(5 x^{2}-3 x+1\right) \geq 0, \quad f_{4}(x, 0,0)=10 x^{4} \geq 0 .
$$

Proposition 4.2. If $x, y, z$ are real numbers, then

$$
\sum \frac{1}{x^{2}+7\left(y^{2}+z^{2}\right)} \leq \frac{9}{5(x+y+z)^{2}}
$$

with equality for $x=y=z$, and for $x / 4=y=z[9]$.

Proof. Let $p=x+y+z$ and $q=x y+y z+z x$. Write the inequality as $f_{6}(x, y, z) \geq 0$, where

$$
f_{6}(x, y, z)=9 \prod\left(x^{2}+7 y^{2}+7 z^{2}\right)-5 p^{2} \sum\left(7 x^{2}+y^{2}+7 z^{2}\right)\left(7 x^{2}+7 y^{2}+z^{2}\right) .
$$

Since

$$
\prod\left(x^{2}+7 y^{2}+7 z^{2}\right)=\prod\left[7\left(p^{2}-2 q\right)-6 x^{2}\right],
$$

$f_{6}(x, y, z)$ has the highest coefficient $A=9(-6)^{3}<0$. By Theorem 2.3 , it suffices to prove the desired inequality for $y=z=1$ and for $y=z=0$. Indeed, we have

$$
f_{6}(x, 1,1)=18\left(7 x^{2}+8\right)(x-1)^{2}(x-4)^{2} \geq 0, \quad f_{6}(x, 0,0)=126 x^{6} \geq 0 .
$$

Proposition 4.3. If $x, y, z$ are real numbers, then

$$
\sum \frac{(x+y)(x+z)}{x^{2}+4\left(y^{2}+z^{2}\right)} \leq \frac{4}{3}
$$

with equality for $x=y=z$, and for $2 x / 7=y=z$.

Proof. Write the inequality as $f_{6}(x, y, z) \geq 0$, where

$$
\begin{aligned}
f_{6}(x, y, z) & =4 \prod\left(x^{2}+4 y^{2}+4 z^{2}\right) \\
& -3 \sum(x+y)(x+z)\left(4 x^{2}+y^{2}+4 z^{2}\right)\left(4 x^{2}+4 y^{2}+z^{2}\right) .
\end{aligned}
$$

Let $p=x+y+z$ and $q=x y+y z+z x$. From

$$
\begin{aligned}
f_{6}(x, y, z) & =4 \prod\left(4 p^{2}-8 q-3 x^{2}\right) \\
& -3 \sum\left(x^{2}+q\right)\left(4 p^{2}-8 q-3 y^{2}\right)\left(4 p^{2}-8 q-3 z^{2}\right),
\end{aligned}
$$

it follows that $f_{6}$ has the highest coefficient $A=4(-3)^{3}-3^{3}<0$. By Theorem 2.3, it suffices to prove the desired inequality for $y=z=1$ and for $y=z=0$. We have

$$
f_{6}(x, 1,1)=\left(4 x^{2}+5\right)(x-1)^{2}(2 x-7)^{2} \geq 0, \quad f_{6}(x, 0,0)=16 x^{6} \geq 0 .
$$


Proposition 4.4. If $x, y, z$ are real numbers such that $x+y+z=3$, then

$$
\sum \frac{1}{8+5\left(y^{2}+z^{2}\right)} \leq \frac{1}{6}
$$

with equality for $x=y=z$, and for $x / 13=y=z[10]$.

Proof. Let $p=x+y+z$ and $q=x y+y z+z x$. Write the inequality in the homogeneous form

$$
\sum \frac{3}{8 p^{2}+45\left(y^{2}+z^{2}\right)} \leq \frac{1}{2 p^{2}}
$$

which is equivalent to $f_{6}(x, y, z) \geq 0$, where

$$
f_{6}(x, y, z)=\prod\left(53 p^{2}-90 q-45 x^{2}\right)-6 p^{2} \sum\left(53 p^{2}-90 q-45 y^{2}\right)\left(53 p^{2}-90 q-45 z^{2}\right) .
$$

Clearly, $f_{6}$ has the highest coefficient $A=(-45)^{3}$. Since $A<0$, it suffices to prove the desired inequality for $y=z$ (see Theorem 2.3). In this case, the original inequality is equivalent to the obvious inequality

$$
(x-1)^{2}(x-13)^{2} \geq 0 .
$$

Proposition 4.5. Let $x, y, z$ be real numbers. If $k \geq 2$, then

$$
\sum \frac{k(k-3) x^{2}+2(k-1) y z}{k x^{2}+y^{2}+z^{2}} \leq \frac{3(k+1)(k-2)}{k+2},
$$

with equality for $x=y=z$, and for $k x / 2=y=z$ [11].

Proof. Setting $m=\frac{k(k-3)}{2(k-1)}$, we have to prove that $f_{6}(x, y, z) \geq 0$, where

$$
\begin{aligned}
f_{6}(x, y, z)= & 3(m+1) \prod\left(k x^{2}+y^{2}+z^{2}\right) \\
& -(k+2) \sum\left(m x^{2}+y z\right)\left(x^{2}+k y^{2}+z^{2}\right)\left(x^{2}+y^{2}+k z^{2}\right) .
\end{aligned}
$$

Let $p=x+y+z, q=x y+y z+z x, r=x y z$. From

$$
\begin{aligned}
f_{6}(x, y, z)= & 3(m+1) \prod\left[p^{2}-2 q+(k-1) x^{2}\right] \\
& -(k+2) \sum\left(m x^{2}+y z\right)\left[p^{2}-2 q+(k-1) y^{2}\right]\left[p^{2}-2 q+(k-1) z^{2}\right],
\end{aligned}
$$

it follows that $f_{6}(x, y, z)$ has the same highest coefficient as

$$
\begin{aligned}
f(x, y, z) & =3(m+1)(k-1)^{3} r^{2}-(k+2)(k-1)^{2} \sum y^{2} z^{2}\left(m x^{2}+y z\right) \\
& =3(k-1)^{2}[(k-1)(m+1)-(k+2) m] r^{2}-(k+2)(k-1)^{2} \sum y^{3} z^{3} .
\end{aligned}
$$

Therefore,

$$
A=3(k-1)^{2}[(k-1)(m+1)-(k+2) m-(k+2)]=\frac{9\left(k^{2}-1\right)(2-k)}{2} .
$$

We see that $A \leq 0$ for $k \geq 2$. By Theorem 2.3, the inequality $f_{6}(x, y, z) \geq 0$ holds if and only if $f_{6}(x, 1,1) \geq 0$ and $f_{6}(x, 0,0) \geq 0$ for all real $x$. Indeed,

$$
f_{6}(x, 1,1)=\left(x^{2}+k+1\right)(x-1)^{2}(k x-2)^{2} \geq 0, \quad f_{6}(x, 0,0)=k^{2} x^{6} \geq 0 .
$$


Proposition 4.6. If $x, y, z$ are real numbers, then

$$
\sum(x-y)(x-z)(x-2 y)(x-2 z)(x-3 y)(x-3 z) \geq 3(x-y)^{2}(y-z)^{2}(z-x)^{2},
$$

with equality for $x=y=z$, for $x / 2=y=z$, for $x / 3=y=z$, and for $x=0$ and $y+z=0$ [4].

Proof. Write the inequality in the form $f_{6}(x, y, z) \geq 0$, and apply Theorem 2.4. Using (1.2)...1.6. we get

$$
\begin{gathered}
\sum(x-y)(x-z)(x-2 y)(x-2 z)(x-3 y)(x-3 z)= \\
=\sum x^{6}-6 \sum x y\left(x^{4}+y^{4}\right)+11 \sum x^{2} y^{2}\left(x^{2}+y^{2}\right)+24 \sum x^{3} y^{3} \\
+36 x y z \sum x^{3}-96 x y z \sum x y(x+y)+363 x^{2} y^{2} z^{2} \\
=819 r^{2}+26\left(p^{3}-11 p q\right) r+p^{6}-12 p^{4} q+44 p^{2} q^{2}-12 q^{3} .
\end{gathered}
$$

Using now (1.9), we can write $f_{6}(x, y, z)$ in the form (1.7), where

$$
A=900, \quad g_{1}(p, q)=2\left(19 p^{3}-170 p q\right), \quad g_{2}(p, q)=p^{6}-12 p^{4} q+41 p^{2} q^{2} .
$$

The condition (a) in Theorem 2.4 is fulfilled since

$$
f_{6}(t, 1,1)=(t-1)^{2}(t-2)^{2}(t-3)^{2} \geq 0, \quad f_{6}(t, 0,0)=t^{6} \geq 0 .
$$

We will show that the condition (c) in Theorem 2.4 is also fulfilled. We have

$$
d(p, q)=g_{1}^{2}(p, q)-4 A g_{2}(p, q)=-4 p^{2}\left(539 p^{4}-4340 p^{2} q+8000 q^{2}\right) .
$$

Since $d(p, q)>0$ yields $p^{2}<6 q$, it follows that

$$
d\left(1+2 t, 2 t+t^{2}\right)>0 \Rightarrow 2 t^{2}+8 t-1>0 \Rightarrow t \in(-\infty,-2) \cup\left(\frac{1}{9}, \infty\right),
$$

and hence

$$
\Gamma_{2} \subset(-\infty,-2) \cup\left(\frac{1}{9}, \infty\right) .
$$

The condition (c) is fulfilled if $h_{2}(t) \leq 0$ for $t \in[-1 / 2,1] \cap \Gamma_{2}=(1 / 9,1]$. Indeed,

$$
\begin{aligned}
h_{2}(t) & =2 A t^{2}+g_{1}\left(1+2 t, 2 t+t^{2}\right) \\
& =2\left[900 t^{2}+19(1+2 t)^{3}-170(1+2 t)\left(2 t+t^{2}\right)\right] \\
& <20\left[90 t^{2}+2(1+2 t)^{3}-17(1+2 t)\left(2 t+t^{2}\right)\right] \\
& =20\left(-18 t^{3}+29 t^{2}-22 t+2\right) \\
& <40\left(-5 t^{3}+15 t^{2}-11 t+1\right) \\
& =40(1-t)\left(1-10 t+5 t^{2}\right) \leq 0 .
\end{aligned}
$$

Proposition 4.7. If $x, y, z$ are real numbers, then

$$
\sum x^{2}(x-y)(x-z) \geq \frac{2(x-y)^{2}(y-z)^{2}(z-x)^{2}}{x^{2}+y^{2}+z^{2}}
$$

with equality for $x=y=z$, and for $x=0$ and $y=z$ [5]. 
Proof. Write the inequality as $f_{6}(x, y, z) \geq 0$, where

$$
f_{6}(x, y, z)=\left(x^{2}+y^{2}+z^{2}\right) \sum x^{2}(x-y)(x-z)-2(x-y)^{2}(y-z)^{2}(z-x)^{2},
$$

and apply Theorem 2.4. Using 1.9 and the identities

$$
\begin{aligned}
\sum x^{2}(x-y)(x-z) & =\sum x^{4}-\sum x y\left(x^{2}+y^{2}\right)+x y z \sum x \\
& =6 p r+p^{4}-5 p^{2} q+4 q^{2}
\end{aligned}
$$

we can write $f_{6}(x, y, z)$ in the form 1.7$)$, where

$$
A=54, \quad g_{1}(p, q)=2\left(7 p^{3}-24 p q\right), \quad g_{2}(p, q)=p^{6}-7 p^{4} q+12 p^{2} q^{2} .
$$

The condition (a) in Theorem 2.4 is fulfilled since

$$
f_{6}(t, 1,1)=\left(t^{2}+2\right) t^{2}(t-1)^{2} \geq 0, \quad f_{6}(t, 0,0)=t^{6} \geq 0 .
$$

We will show that the condition (b) in Theorem 2.4 is also fulfilled. We have

$$
d(p, q)=g_{1}^{2}(p, q)-4 A g_{2}(p, q)=4 p^{2}\left(5 p^{2}-12 q\right)\left(6 q-p^{2}\right) .
$$

Since $d(p, q)>0$ is equivalent to $6 q>p^{2}$, we get

$$
d(t+2,2 t+1)>0 \Longleftrightarrow 6(2 t+1)>(t+2)^{2} \Longleftrightarrow \Gamma_{1}=(4-3 \sqrt{2}, 4+3 \sqrt{2}),
$$

and hence

$$
[-2,1] \cap \Gamma_{1}=(4-3 \sqrt{2}, 1] .
$$

Since $4-3 \sqrt{2}>-1 / 4$, it suffices to show that $h_{1}(t) \geq 0$ for $t \in[-1 / 4,1]$. We have

$$
h_{1}(t)=2 A t+g_{1}(t+2,2 t+1)=2\left(7 t^{3}-6 t^{2}+18 t+8\right) .
$$

Clearly, $h_{1}(t)>0$ for $t \geq 0$. Also, $h_{1}(t)>0$ for $t \in[-1 / 4,0)$, since

$$
7 t^{3}-6 t^{2}+18 t+8>8 t^{3}-6 t^{2}+30 t+8=2(4 t+1)\left(t^{2}-t+4\right) \geq 0 .
$$

Proposition 4.8. Let $x, y, z$ be real numbers. If $-1 / 2 \leq k \leq 1$, then

$$
4 \sum y z(x-y)(x-z)(x-k y)(x-k z)+(x-y)^{2}(y-z)^{2}(z-x)^{2} \geq 0,
$$

with equality for $x=y=z$, for $y=z=0$, and for $x / k=y=z, k \neq 0$. If $k=0$, then equality occurs also for $x=0$ and $y=z[[6]$.

Proof. Denote the left-hand side of the inequality by $f_{6}(x, y, z)$. From 1.8$)$ and

$$
\begin{gathered}
\sum y z(x-y)(x-z)(x-k y)(x-k z)=k^{2} \sum x^{3} y^{3}+x y z \sum x^{3} \\
-\left(k^{2}+k+1\right) x y z \sum x y(x+y)+3(k+1)^{2} x^{2} y^{2} z^{2},
\end{gathered}
$$

we get

$$
\begin{gathered}
f_{6}(x, y, z)=\sum x^{2} y^{2}\left(x^{2}+y^{2}\right)+2\left(2 k^{2}-1\right) \sum x^{3} y^{3}+2 x y z \sum x^{3} \\
-2\left(2 k^{2}+2 k+1\right) x y z \sum x y(x+y)+6\left(2 k^{2}+4 k+1\right) x^{2} y^{2} z^{2} .
\end{gathered}
$$


Using (1.2) ...1.6), we can write $f_{6}(x, y, z)$ in the form 1.7), where

$$
A=9(2 k+1)^{2}, \quad g_{1}(p, q)=-2\left(8 k^{2}+2 k-1\right) p q, \quad g_{2}(p, q)=p^{2} q^{2}+4\left(k^{2}-1\right) q^{3} .
$$

For $k=-1 / 2$, we have $f_{6}(x, y, z)=q^{2}\left(p^{2}-3 q\right) \geq 0$, and for $k=1$, we have $f_{6}(x, y, z)=(p q-9 r)^{2} \geq 0$. Then, we consider further that $-1 / 2<k<1$. Since $A>0$, we apply Theorem 2.4. The condition (a) in Theorem 2.4 is fulfilled since

$$
f_{6}(t, 1,1)=4(t-1)^{2}(t-k)^{2} \geq 0, \quad f_{6}(t, 0,0)=0 .
$$

To complete the proof, we will show that the condition (b) in Theorem 2.4 is fulfilled. We have

$$
\begin{gathered}
d(p, q)=16(1-k)(1+2 k)^{2} q^{2}\left[9(k+1) q-2(2 k+1) p^{2}\right], \\
d(p, q)>0 \Longleftrightarrow 9(k+1) q-2(2 k+1) p^{2}>0, \\
d(t+2,2 t+1)>0 \Longleftrightarrow 2(2 k+1) t^{2}-2(k+5) t+7 k-1<0, \\
\Gamma_{1}=\left(\frac{k+5-3 \sqrt{3\left(1-k^{2}\right)}}{2(2 k+1)}, \frac{k+5+3 \sqrt{3\left(1-k^{2}\right)}}{2(2 k+1)}\right), \\
{[-2,1] \cap \Gamma_{1}=\left(t_{1}, 1\right],}
\end{gathered}
$$

where

$$
t_{1}=\frac{k+5-3 \sqrt{3\left(1-k^{2}\right)}}{2(2 k+1)}>\frac{-1}{2} .
$$

We need to show that $h_{1}(t) \geq 0$ for $t_{1}<t \leq 1$, where

$$
h_{1}(t)=2 A t+g_{1}(t+2,2 t+1)=18(2 k+1)^{2} t-2(4 k-1)(2 k+1)(t+2)(2 t+1) .
$$

This is true if $h(t) \geq 0$ for $t_{1}<t \leq 1$, where

$$
h(t)=9(2 k+1) t-(4 k-1)(t+2)(2 t+1),
$$

with

$$
h^{\prime}(t)=4(1-4 k) t+14-2 k .
$$

Since $h^{\prime}(-1 / 2)=6(k+2)>0$ and $h^{\prime}(1)=18(1-k)>0$, we have $h^{\prime}(t)>0$ for $-1 / 2 \leq t \leq 1$, and hence $h(t)$ is increasing on $\left[t_{1}, 1\right]$. Therefore, it suffices to show that $h\left(t_{1}\right) \geq 0$. From $2(2 k+1) t_{1}^{2}-2(k+5) t_{1}+7 k-1=0$, we get

$$
h\left(t_{1}\right)=\frac{3(1-k)\left[4(2+k) t_{1}+1-4 k\right]}{2 k+1} .
$$

Thus, we need to show that $4(2+k) t_{1}+1-4 k \geq 0$, which can be written as

$$
7+4 k-2 k^{2} \geq 3(2+k) \sqrt{3\left(1-k^{2}\right)} .
$$

By squaring, we get the obvious inequality $(2 k+1)^{4} \geq 0$.

Proposition 4.9. If $x, y, z$ are real numbers, then

$$
16 \sum y z\left(x^{2}-y^{2}\right)\left(x^{2}-z^{2}\right)+5(x-y)^{2}(y-z)^{2}(z-x)^{2} \geq 0,
$$

with equality for $x=y=z$, for $-x=y=z$, and for $y=z=0[6]$. 
Proof. Denote the left-hand side of the inequality by $f_{6}(x, y, z)$ and apply Theorem 2.4. From

$$
\sum y z\left(x^{2}-y^{2}\right)\left(x^{2}-z^{2}\right)=\sum x^{3} y^{3}+x y z \sum x^{3}-x y z \sum x y(x+y)
$$

and 1.8$)$, we have

$$
\begin{aligned}
f_{6}(x, y, z) & =5 \sum x^{2} y^{2}\left(x^{2}+y^{2}\right)+6 \sum x^{3} y^{3}+6 x y z \sum x^{3} \\
& -6 x y z \sum x y(x+y)-30 x^{2} y^{2} z^{2} .
\end{aligned}
$$

Using (1.2)...11.6), we can write $f_{6}(x, y, z)$ in the form (1.7), where

$$
A=9, \quad g_{1}(p, q)=-2\left(2 p^{3}+11 q\right), \quad g_{2}(p, q)=5 p^{2} q^{2}-4 q^{3} .
$$

The condition (a) in Theorem 2.4 is fulfilled since

$$
f_{6}(t, 1,1)=16\left(t^{2}-1\right)^{2} \geq 0, \quad f_{6}(t, 0,0)=0 .
$$

To complete the proof, we will show that the condition (c) in Theorem 2.4 is fulfilled. We have

$$
\begin{gathered}
d(p, q)=g_{1}^{2}(p, q)-4 A g_{2}(p, q)=16\left(p^{2}+q\right)^{2}\left(p^{2}+9 q\right), \\
d(p, q)>0 \Longleftrightarrow p^{2}+9 q>0, \\
d\left(1+2 t, 2 t+t^{2}\right)>0 \Longleftrightarrow 13 t^{2}+22 t+1>0, \\
\Gamma_{2}=\left(-\infty, \frac{-11-6 \sqrt{3}}{13}\right) \cup\left(\frac{-11+6 \sqrt{3}}{13}, \infty\right), \\
{\left[\frac{-1}{2}, 1\right] \cap \Gamma_{2}=\left(\frac{-11+6 \sqrt{3}}{13}, 1\right]}
\end{gathered}
$$

We need to show that $h_{2}(t) \leq 0$ for $\frac{-11+6 \sqrt{3}}{13}<t \leq 1$. Indeed,

$$
\begin{aligned}
h_{2}(t)=2 A t^{2}+g_{1}\left(1+2 t, 2 t+t^{2}\right) & =18 t^{2}-4(1+2 t)^{3}-22(1+2 t)\left(2 t+t^{2}\right) \\
& =-4(t+1)\left(19 t^{2}+16 t+1\right)<0 .
\end{aligned}
$$

Proposition 4.10. Let $x, y, z$ be real numbers. If $1 \leq k \leq 4$, then

$$
\sum x^{2}(x-y)(x-z)(x-k y)(x-k z) \geq(5-3 k)(x-y)^{2}(y-z)^{2}(z-x)^{2},
$$

with equality for $x=y=z$, for $x=0$ and $y=z$, and for $x / k=y=z$ [7].

Proof. Denote the left-hand side of the inequality by $f(x, y, z)$ and write the desired inequality as $f_{6}(x, y, z) \geq$ 0 . Using (1.2) ...11.6), we have

$$
\begin{aligned}
f(x, y, z)= & \sum x^{6}-(k+1) \sum x y\left(x^{4}+y^{4}\right)+k \sum x^{2} y^{2}\left(x^{2}+y^{2}\right) \\
& +(k+1)^{2} x y z \sum x^{3}-k(k+1) x y z \sum x y(x+y)+3 k^{2} x^{2} y^{2} z^{2}, \\
f(x, y, z)= & 9\left(k^{2}+k+1\right) r^{2}+\left[\left(k^{2}+k+8\right) p^{3}-2\left(2 k^{2}+5 k+11\right) p q\right] r+p^{6} \\
& -(k+7) p^{4} q+(5 k+13) p^{2} q^{2}-4(k+1) q^{3} .
\end{aligned}
$$


Using then 1.9$)$, we can write $f_{6}(x, y, z)$ in the form 1.7$)$, where

$$
A=9(k-4)^{2}, \quad g_{1}=(4-k)(7-k) p\left(p^{2}-4 q\right), \quad g_{2}=\left(p^{2}-4 q\right)^{2}\left[p^{2}-(k-1) q\right] .
$$

For $k=1$, we have $f_{6}(x, y, z)=\left(9 r-p^{3}+4 p q\right)^{2} \geq 0$, and for $k=4$, we have $f_{6}(x, y, z)=\left(p^{2}-4 q\right)^{2}\left(p^{2}-3 q\right) \geq$ 0 . Then, we consider further that $1<k<4$. Since $A>0$, we apply Theorem 2.4. The condition (a) in Theorem 2.4 is fulfilled since

$$
f_{6}(t, 1,1)=t^{2}(t-1)^{2}(t-k)^{2} \geq 0, \quad f_{6}(t, 0,0)=t^{6} \geq 0 .
$$

We will show that the condition (c) is satisfied. We have

$$
d(p, q)=g_{1}^{2}-4 A g_{2}=(k-1)(4-k)^{2}\left(p^{2}-4 q\right)^{2}\left[36 q-(13-k) p^{2}\right] .
$$

Since $d(p, q)>0$ is equivalent to $36 q>(13-k) p^{2}$, we get

$$
d\left(1+2 t, 2 t+t^{2}\right)>0 \Longleftrightarrow k(1+2 t)^{2}>16 t^{2}-20 t+13 .
$$

The condition (c) is satisfied if $h_{2}(t) \leq 0$ for $k(1+2 t)^{2}>16 t^{2}-20 t+13$. Since

$$
\begin{aligned}
h_{2}(t) & =2 A t^{2}+g_{1}\left(1+2 t, 2 t+t^{2}\right) \\
& =(4-k)\left[16 t^{2}-14 t+7-k\left(10 t^{2}-2 t+1\right)\right],
\end{aligned}
$$

we need to show that $k\left(10 t^{2}-2 t+1\right) \geq 16 t^{2}-14 t+7$. Indeed,

$$
\begin{aligned}
k\left(10 t^{2}-2 t+1\right) & -16 t^{2}+14 t-7> \\
& >\frac{\left(16 t^{2}-20 t+13\right)\left(10 t^{2}-2 t+1\right)}{(1+2 t)^{2}}-16 t^{2}+14 t-7 \\
& =\frac{6(t-1)^{2}(4 t-1)^{2}}{(1+2 t)^{2}} \geq 0 .
\end{aligned}
$$

Proposition 4.11. If $x, y, z$ are real numbers, then

$$
\sum x^{2}(x-y)(x-z)(x+3 y)(x+3 z)+\frac{133}{64}(x-y)^{2}(y-z)^{2}(z-x)^{2} \geq 0,
$$

with equality for $x=y=z$, for $x=0$ and $y=z$, and for $-x / 3=y=z[7]$.

Proof. Let us denote

$$
f(x, y, z)=\sum x^{2}(x-y)(x-z)(x+3 y)(x+3 z),
$$

and write the inequality as $f_{6}(x, y, z) \geq 0$, where

$$
f_{6}(x, y, z)=64 f(x, y, z)+133(x-y)^{2}(y-z)^{2}(z-x)^{2} \geq 0 .
$$

Applying 4.1) for $k=-3$, we get

$$
f(x, y, z)=63 r^{2}+14\left(p^{3}-2 p q\right) r+p^{6}-4 p^{4} q-2 p^{2} q^{2}+8 q^{3} .
$$

Using then (1.9), we can write $f_{6}(x, y, z)$ in the form (1.7), where

$$
A=441, \quad g_{1}=14 p\left(26 p^{2}+43 q\right), \quad g_{2}=\left(p^{2}-4 q\right)\left(64 p^{4}+5 q^{2}\right) .
$$


The condition (a) in Theorem 2.4 is fulfilled since

$$
f_{6}(t, 1,1)=64 t^{2}(t-1)^{2}(t+3)^{2} \geq 0, \quad f_{6}(t, 0,0)=64 t^{6} \geq 0 .
$$

We will show that the condition (b) is satisfied. We have

$$
d(p, q)=g_{1}^{2}-4 A g_{2}=25600\left(p^{2}+2 q\right)^{2}\left(p^{2}+45 q\right) .
$$

Since $d(p, q)>0$ is equivalent to $p^{2}+45 q>0$, we get

$$
\begin{gathered}
d(t+2,2 t+1)>0 \Longleftrightarrow t^{2}+94 t+49>0, \\
\Gamma_{1}=\left(-\infty, t_{1}\right) \cup\left(t_{2}, \infty\right), \quad t_{1} \approx-93.47, t_{2} \approx-0.524, \\
{[-2,1] \cap \Gamma_{1}=\left(t_{2}, 1\right] .}
\end{gathered}
$$

Therefore, we need to show that $h_{1}(t) \geq 0$ for $t \in\left(t_{2}, 1\right]$. Indeed,

$$
h_{1}(t)=2 A t+g_{1}(t+2,2 t+1)=28(t+3)\left(13 t^{2}+82 t+49\right)>0,
$$

since $13 t^{2}+82 t+49 \geq 82 t+49>0$.

\section{References}

[1] T. Ando, Some Homogeneous Cyclic Inequalities of Three Variables of Degree Three and Four, The Australian Journal of Mathematical Analysis and Applications, vol. 7, issue 2, art. 12, 2011. 1

[2] V. Cirtoaje, On the Cyclic Homogeneous Polynomial Inequalities of Degree Four, Journal of Inequalities in Pure and Applied Mathematics, vol. 10, issue 3, art. 67, 2009 [ONLINE/http://www.emis.de/journals/JIPAM/ article1123.html 1

[3] V. Cirtoaje, Algebraic Inequalities - Old and New Methods, GIL Publishing House, 2006. 11

[4] Art of Problem Solving Forum, May, 2009, [ONLINE: http://www.artofproblemsolving.com/Forum/viewtopic. php?t=278972 $1,4.6$

[5] Art of Problem Solving Forum, August, 2009, [ONLINE: http://www.artofproblemsolving.com/Forum/ viewtopic . php?t=297720 $1,4.7$

[6] Art of Problem Solving Forum, August, 2010, [ONLINE: http://www.artofproblemsolving.com/Forum/ viewtopic $\cdot$ php?t=363235, $1,4.8,4.9$

[7] Art of Problem Solving Forum, July, 2009, [ONLINE: http://www.artofproblemsolving.com/Forum/viewtopic . $\mathrm{php} ? \mathrm{t}=284163 \&$ start $=20$, $1,4.10,4.11$

[8] Art of Problem Solving Forum, April, 2008, [ONLINE: http://www.artofproblemsolving.com/Forum/ viewtopic . php?t=272416 4.1

[9] Art of Problem Solving Forum, Mars, 2008, [ONLINE: http://www.artofproblemsolving.com/Forum/ viewtopic.php?t=192206 4.2

[10] Art of Problem Solving Forum, May, 2009, [ONLINE: http://www.artof problemsolving.com/Forum/viewtopic. php?t=275815 4.4

[11] Art of Problem Solving Forum, February, 2008, [ONLINE: http://www.artofproblemsolving.com/Forum/ viewtopic.php?t=186265 